\title{
Unambiguous Wide-Range Optical Voltage Sensor with Dual Operating Wavelengths
}

\author{
Xun Gu${ }^{1}$, Sergio V. Marchese ${ }^{1}$, Klaus Bohnert ${ }^{1}$ \\ ${ }^{1}$ ABB Switzerland Corporate Research Center, Segelhofstrasse 1K, 5405 Baden-Dättwil, Switzerland \\ xun.gu@ch.abb.com
}

\begin{abstract}
:
We extend the unambiguous measurement range of an electro-optic voltage sensor from $75 \mathrm{kV}$ to $100 \mathrm{~s}$ of kilovolts (spanning multiple $2 \pi$ phase shift ranges), by combining phase shift principal value measurements at two different wavelengths. Compared with previous two-wavelength techniques, this new approach completely eliminates all remaining measurement ambiguities.
\end{abstract}

Key words: phase shift measurement, two-wavelength measurement, electro-optic voltage sensor, period disambiguation.

\section{Introduction}

There has been great interest in the electric power industry in developing new voltage sensors based on optical measurement principles. Compared with conventional technologies, the optical sensors are compact, galvanically insulated, insusceptible to electromagnetic interference, and cost-effective. For emerging HVDC applications (e.g. offshore wind parks and long-distance power transmission), optical voltage sensors are even more attractive, as conventional capacitive and inductive transformers cannot measure DC voltages.

Optical voltage sensors rely on the interference between two orthogonal polarization modes. The applied voltage induces a relative phase shift, which is measured by photodetectors. For an electro-optic voltage sensor, bismuth germanate $\left(\mathrm{Bi}_{4} \mathrm{Ge}_{3} \mathrm{O}_{12}\right.$, or $\left.\mathrm{BGO}\right)$ of the $\overline{4} 3 m$ symmetry class is a preferred sensing medium. Particularly advantageous is the longitudinal configuration, in which the [001] crystal axis is oriented along the optical path so that the measured phase shift is proportional to the path integral of the electric field $\int \boldsymbol{E} \cdot d \boldsymbol{s}$, i.e., the applied voltage between the crystal end faces. The field integration produces an accurate measurement of the voltage, irrespective of space charge and local field distributions.

Typically, an electro-optic voltage sensor uses the sensing crystal together with some polarizers and waveplates [1]. The output of such a polarimetric sensor is a sinusoidal function of the differential phase shift between two orthogonal polarizations. Phase shifts of $\phi$ and $\phi+2 n \pi(n$ being an integer number) produce the same polarimetric output, and hence cannot be distinguished from one another. (For an individual polarimetric signal, there is an additional sign ambiguity within a single period, i.e., $\cos \phi=\cos (-\phi)$, which can be removed by adding a quadrature signal $\sin \phi$.)

The periodwise ambiguity is an inherent problem for all interferometric measurements. For relative measurements, the measurement range can be extended by fringe-counting or similar historytracking techniques. In AC voltage measurements, one can thus extend the measurement range to many times the $\pi$-voltage (75 kV at $1310 \mathrm{~nm}$ for BGO) by combining quadrature polarimetric signals and using zerocrossing counting [2, 3], facilitated by the fact that the $\mathrm{AC}$ voltage continuously oscillates about zero.

On the other hand, for absolute measurements without history information, the periodwise ambiguity places a fundamental limit to the achievable unambiguous measurement range. Such is the case for the measurement of the DC voltage, which lacks an oscillating waveform and thus a zero voltage reference. Furthermore, the lack of zero reference also makes it difficult to distinguish voltage drifts from effects such as changing optical loss, stress-induced birefringence, etc. Kurosawa and Yoshida attempted to address the drift problem by chopping the applied voltage [4], but such solutions are not amicable to HV applications. Therefore, a wide-range (100s of kilovolts) optical DC voltage sensor calls for a different solution. 


\section{Deficiencies of previous dual-wavelength period disambiguation techniques}

One way to extend the unambiguous range of a phase shift measurement is to combine it with another unambiguous (but coarser) measurement. For some applications, one can simply use two types of sensing medium for high- and low-resolution measurements, respectively [5]. This is however not an option for voltage sensing, due to the scarcity of suitable sensing crystals of different sensitivities.

It has been suggested $[6,7]$ to solve the periodwise ambiguity by combining measurements at two wavelengths of different voltage sensitivities. Although the measurement at either wavelength is still periodic, the dualwavelength pair as a whole generally does not have a periodic dependence on the voltage, and hence can be used to unambiguously allocate the combined sensor's output in a range much larger than either sensor's unambiguous range. The dual-wavelength sensor outputs are then

$$
\begin{gathered}
y_{1}(V)=\cos \phi_{1}=\cos \left(q_{1} V+\delta_{1}\right) \\
\quad \text { and } \\
y_{2}(V)=\cos \phi_{2}=\cos \left(q_{2} V+\delta_{2}\right)
\end{gathered}
$$

where $q_{1} \neq q_{2}$ are the voltage sensitivities of the electro-optic phase shifts $\phi_{1,2}$ at the two wavelengths $\lambda_{1,2}$, and $\delta_{1,2}$ are the phase offsets (set to 0 in later simulations). For the BGO voltage sensor, $q=2 \pi n^{3} r_{41} / \lambda$, where $n$ is the refractive index, and $r_{41}$ is the electro-optic coefficient. For simplicity and without loss of generality, here the amplitudes of the outputs $y_{1}$ and $y_{2}$ are set to 1 , and any potential offsets to 0 .

While the dual-wavelength period disambiguation seems straightforward at the first glance, an important caveat was disregarded in previous publications. It becomes evident when the pair $Y(V)=\left[y_{1}(V), y_{2}(V)\right]$ is plotted as the voltage changes, resulting in a Lissajous figure (see Fig. 1a). The shape of a Lissajous figure is characteristic of the ratio $q_{1} / q_{2}$ as well as the phase offset difference $\delta_{1}-\delta_{2}$. Therefore, it is widely used to visually represent the relationship between harmonic signals.

The Lissajous figure maps a 1D variable to a point along the trace $Y(V)$ in the 2D $y_{1}-y_{2}$ plane. If the ratio $q_{1} / q_{2}$ is rational, i.e., $q_{1} / q_{2}=N_{1} / N_{2}$, where $N_{1}$ and $N_{2}$ are integers, the trace is a closed curve, and the $2 \mathrm{D}$ period

$$
d=2 \pi N_{1} / q_{1}=2 \pi N_{2} / q_{2}
$$

is increased $N_{i}$-fold from the singlemeasurement period $2 \pi / q_{i}$. If $q_{1} / q_{2}$ is irrational, the trace is not closed, meaning the two- wavelength measurement $Y(V)$ is aperiodic. Therefore, one can use the dual-wavelength method to significantly increase the unambiguous range of the polarimetric measurement.

It is also obvious from the Lissajous figure that the trace makes many crossings upon itself as the voltage progresses. At any crossing point in the figure, there exist two possible voltage values that produce the same sensor output $Y(V)$. Therefore, one crossing point corresponds to a pair of two possible voltage values that cannot be mutually distinguished in the measurement.

One solution to this problem has been proposed by adding a third measurement at a different wavelength [7]. The trace in the 3D space $Y(V)=\left[y_{1}(V), y_{2}(V), y_{3}(V)\right]$, known as a Lissajous knot, generally does not make crossings upon itself as the voltage progresses (with the exception of some isolated degenerate cases). Therefore, no ambiguity generally exists. However, operating light sources, detectors and other optical components at three or more wavelengths increases the complexity and cost, and reduces the reliability of the entire sensor system. Furthermore, mapping a 3D (or higherdimensional) measurement to a single measurand value also involves more complicated signal processing. Therefore, it is not a preferred approach to solve the ambiguity problem.

The properties of this ambiguity can be better studied by performing an arccos transformation on the Lissajous figure.

$$
Z=\left[z_{1}, z_{2}\right]=\left[\arccos y_{1}, \arccos y_{2}\right]
$$

where arccos returns the principal value of the inverse cosine function, defined in the range [0, $\pi]$. Note that since the arccos transformation is a bijective (invertible one-to-one) mapping from $[-1,1]$ to $[0, \pi], Y$ and $Z$ are equivalent representations of the same data. Making use of the equality $\arccos (\cos \phi)=|p v \phi|$ results in:

$$
Z=\left[z_{1}, z_{2}\right]=\left[\left|\mathrm{pv}\left(q_{1} V+\delta_{1}\right)\right|,\left|\mathrm{pv}\left(q_{2} V+\delta_{2}\right)\right|\right]
$$

where the function pv yields the principal value of a phase angle defined in the range $(-\pi, \pi]$.

Because $z_{1}$ and $z_{2}$ are each segmented linear functions of $V$, the $Z$ trace, plotted in Fig. $1 \mathrm{~b}$, consists of a series of straight lines, and the voltage $V$ is uniformly distributed along these lines. The entire $Z$ trace can be viewed as the propagation of a straight ray as it is being continually "reflected" at the four boundaries of a square box, defined by $z_{1,2}=0, \pi$. Therefore, half of the segments have a positive slope $q_{2} / q_{1}$, and the other half a negative one $-q_{2} / q_{1}$. 

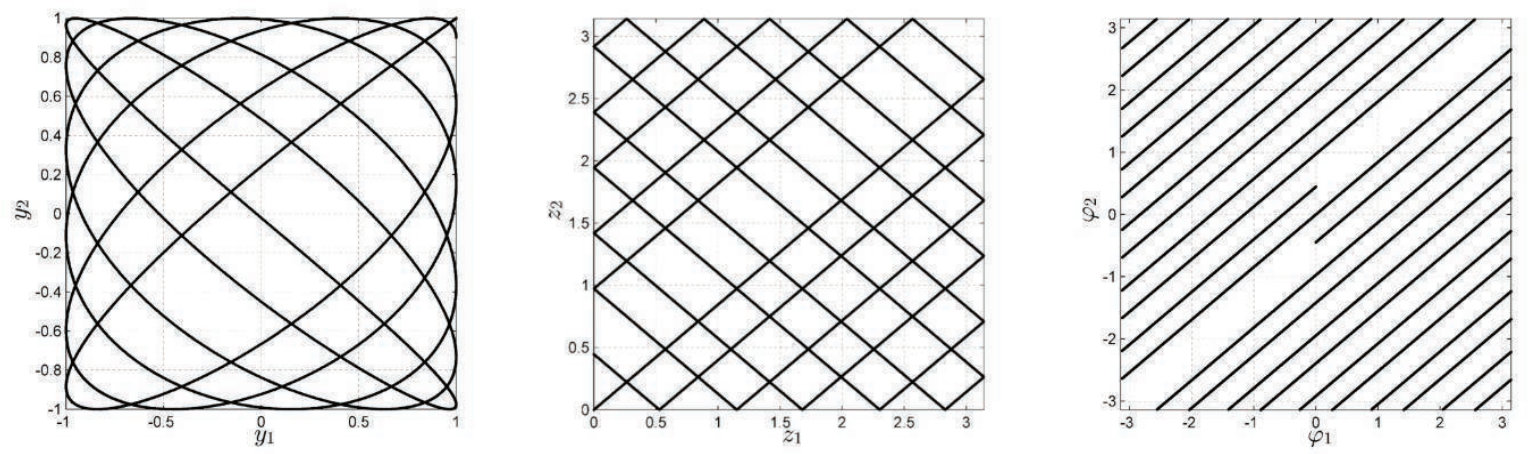

Fig. 1. From left to right: (a) Y trace (Lissajous figure), (b) Z trace, (c) $\Phi$ trace. For these plots, a double-pass $B G O$ voltage sensor is modeled at 1310 and $1550 \mathrm{~nm}$ with $\delta_{1}=\delta_{2}=0$ within [-450kV, 450kV]. Correspondingly in this example, $q_{1}=0.0838 \mathrm{rad} / \mathrm{kV}$, and $q_{1}=0.0708 \mathrm{rad} / \mathrm{kV}$.

Ambiguity is created by the crossing between a positive-slope segment and a negative-slope segment. All the positive-slope segments are parallel to and evenly spaced between each other, as is true also for the negative-slope segments. Consequently, the crossing points are also evenly spaced along the segments. Because the voltage $V$ is uniformly distributed along the straight lines, this also means that the ambiguity points are quasi-uniformly distributed in the entire measurand range.

Some further mathematical analysis will show that the total number of crossing points is roughly $M=N(N-1) / 2$, where $N=\left\lfloor\left(q_{1}+q_{2}\right) L / 2 \pi\right\rfloor$, and $L$ is the size of the measurement range. Obviously, since $N$ is linear with $L$, the total number of ambiguous measurand values $2 M$ increases quasi-quadratically with the measurand range size.

For a BGO voltage sensor, the $\pi$-voltage is about $75 \mathrm{kV}$ at $1310 \mathrm{~nm}$, and $88.7 \mathrm{kV}$ at 1550 $\mathrm{nm}$. If the double-pass polarimetric outputs at these two wavelengths are used, in the voltage range $[0,450 \mathrm{kV}]$, the total number of ambiguous voltage values is 100 , meaning on average one ambiguous voltage value occurs every $4.5 \mathrm{kV}$.

The large number of ambiguity points in a widerange dual-wavelength polarimetric sensor presents a challenge for signal processing. One might argue that, because ambiguity always occurs between two segments of opposite slopes, it is possible to use the time derivative of the measured phase shift to determine on which segment the ambiguous measurement should fall. This approach, however, requires tracking the measurement history, and assumes that the measurand waveform has a meaningfully large local derivative when the ambiguity point occurs. For voltage measurements, this approach might work for AC voltages, but would not work reliably for DC voltages. Furthermore, signal processing is especially problematic for the measurement of fast transient waveforms, in which the sampleto-sample change of the measurand can be larger than the spacing between adjacent ambiguous voltage values.

\section{Complete period disambiguation with $2 \pi-$ range dual-wavelength detection}

Suppose, by a suitable method, the principal values of the phase shifts are determined unambiguously within a $2 \pi$ range, i.e.,

$$
\Phi=\left[\varphi_{1}, \varphi_{2}\right]=\left[\mathrm{pv}\left(q_{1} V+\delta_{1}\right), \mathrm{pv}\left(q_{2} V+\delta_{2}\right)\right]
$$

A plot of $\Phi$ in the $\varphi_{1}-\varphi_{2}$ plane as the voltage $V$ progresses is shown in Fig. 1c. This $\Phi$ trace consists of straight line segments, now all of the same slope $q_{2} / q_{1}$.

The $\Phi$ trace can also be envisioned as the propagation of a ray inside a square box enclosed by boundaries $\varphi_{1,2}= \pm \pi$. When the ray reaches a boundary, it reemerges from the same position on the opposite side, and continues with the same slope. Therefore, all segments in the trace are parallel to each other, and are quasiequally spaced, with no crossing or associated ambiguity points.

Any method that unambiguously determines the phase shift principal value (at each of the wavelengths) can be used for the implementation of this approach. For example, in a quadrature detection scheme, one measures two quadrature signals

$$
\begin{aligned}
& I_{1}=I_{0}(1+A \cos \phi) / 2 \\
& I_{2}=I_{0}(1+A \sin \phi) / 2
\end{aligned}
$$

If the total light power $I_{0}$ is also measured, a complex variable $\mathrm{S}$ can be calculated as

$$
S=\left(\frac{2 I_{1}}{I_{0}}-1\right)+i\left(\frac{2 I_{2}}{I_{0}}-1\right)=A e^{i \phi}
$$


Therefore, the phase shift principal value in the $2 \pi$ range is

$$
\varphi=\operatorname{pv} \phi=\arg S
$$

Other phase measurement techniques, such as the non-reciprocal phase modulation scheme widely used in fiber-optic gyroscopes [8] and current sensors $[9,10]$, may also be used for the dual-wavelength period disambiguation, as long as the phase at each wavelength can be individually determined in a $2 \pi$ range.

A wide-range optical voltage sensor can then be designed using the $2 \pi$-range dual-wavelength period disambiguation method and polarimetric quadrature detection, as shown in Fig. 2.

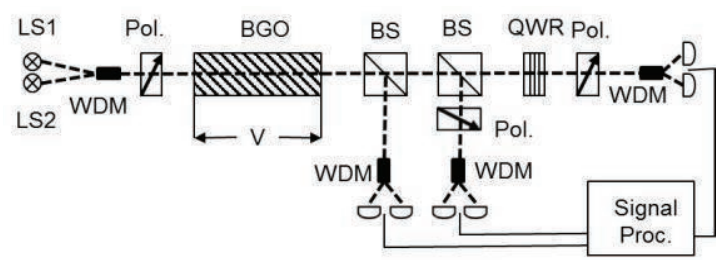

Fig. 2. Schematic of a wide-range unambiguous $B G O$ voltage sensor. LS is light source, WDM wavelengthdivision multiplexer, Pol. polarizer, BS beamsplitter, QWR quarter-wave retarder, $D$ detector.

There are several different approaches with regard to the signal processing procedure of converting the measured dual-wavelength outputs to an unambiguous voltage value.

One approach is the following: After measuring the phase shift principal values $\left[\varphi_{1}, \varphi_{2}\right]$ at the two wavelengths, one generates two lists of possible full values of the phase shifts $\left[\phi_{m}^{(1)}, \phi_{n}^{(2)}\right]=\left[\varphi_{1}+2 m \pi, \varphi_{2}+2 n \pi\right]$ ( $m$ and $n$ are integers) in the measurement range. Then, two lists of possible voltage values are calculated from these phase shifts

$$
\left[V_{m}^{(1)}, V_{n}^{(2)}\right]=\left[\left(\phi_{m}^{(1)}-\delta_{1}\right) / q_{1},\left(\phi_{n}^{(2)}-\delta_{2}\right) / q_{2}\right]
$$

Next, the two lists can be compared to identify a pair of $\left[V_{m}^{(1)}, V_{n}^{(2)}\right]$ with the smallest difference $\left|V_{m}^{(1)}-V_{n}^{(2)}\right|$. Finally, the output can be set as the average of both voltages $\left(V_{m}^{(1)}+V_{n}^{(2)}\right) / 2$.

A second method is the following: Each segment in the $\Phi$ trace can be uniquely labeled with a pair of indices $[m, n]$ by the relationship

$$
\begin{aligned}
{\left[\phi_{1}, \phi_{2}\right]=\left[q_{1} V+\delta_{1}, q_{2} V+\delta_{2}\right] } & \\
& =\left[\varphi_{1}+2 m \pi, \varphi_{2}+2 n \pi\right]
\end{aligned}
$$

Therefore, one can define

$$
\begin{aligned}
\Delta_{m n}=q_{1} \varphi_{2}-q_{2} & \varphi_{1} \\
& =2 \pi\left(m q_{2}-n q_{1}\right) \\
& +\left(q_{1} \delta_{2}-q_{2} \delta_{1}\right)
\end{aligned}
$$

Mathematically, $\Delta_{m n} / \sqrt{q_{1}^{2}+q_{2}^{2}}$ is the signed perpendicular distance from the origin to the segment with the index pair $[m, n]$, with its sign indicating on which side of the origin the segment lies. Therefore, in the entire unambiguous measurement range, each index pair $[m, n]$ corresponds to a unique $\Delta_{m n}$ and vice versa. This mapping can be pre-calculated and saved in a 1D tabular form. An example of the one-to-one correspondence between $\Delta_{m n}$ and $[m, n]$ is shown in Fig. 3.

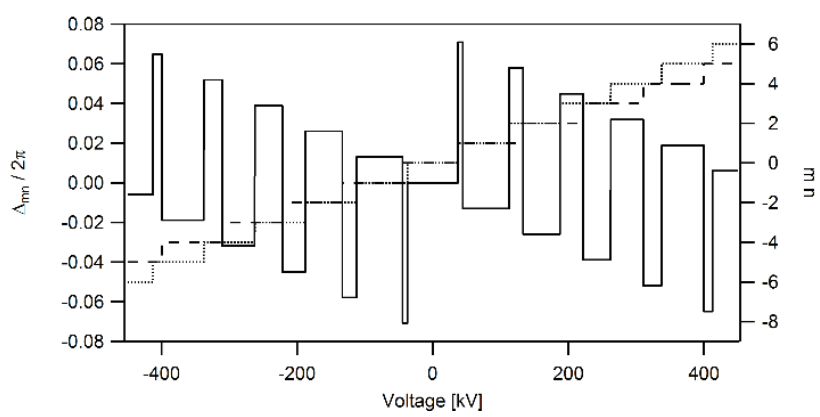

Fig. 3. Segment judgment diagram modeled for a double-pass BGO voltage sensor operating at 1310 $\mathrm{nm}$ and $1550 \mathrm{~nm}$, with $\delta_{1}=\delta_{2}=0$ in a measurement range $[-450 \mathrm{kV}, 450 \mathrm{kV}]$. The solid line is the calculated $\Delta_{m n}$, the dotted line the $q_{1}$ segment number $m$, and the dashed line the $q_{2}$ segment number $n$. Note that there is a one-to-one correspondence between the index pair $[m, n]$ and the $\Delta m n$ value.

Hence, from the measured phase shift principal values $\left[\varphi_{1}, \varphi_{2}\right]$, one can calculate $\Delta_{m n}$ and look up the corresponding indices $[m, n]$ from the precalculated table. Finally, the full values of the phase shift and the corresponding measurand value can be calculated.

The first method involves a search in a $2 \mathrm{D}$ space consisting of two dynamic lists, whereas the second method involves only a 1D lookup in a static list. Therefore, in terms of computational complexity, the second signal processing method is much more efficient.

\section{Guideline for optimal wavelength selection}

We present here a design guideline for the selection of optimal wavelengths for a dualwavelength interferometric sensor.

We start with one given wavelength $\lambda_{1}$ and the corresponding $q_{1}$. For simplicity, it is assumed that $q_{1}=2 \pi N_{1} / L$. Therefore, the measurement at $\lambda_{1}$ results in $N_{1}$ line segment end points on the left (or right) boundary of the $2 \mathrm{D}$ phase space ($\pi / 2, \pi / 2] \times(-\pi / 2, \pi / 2]$.

It is generally advantageous to separate all line segments in Fig. 1c as far away as possible between each other, in order to minimize the influence of measurement noise. This can be 
best realized if the segments uniformly fill the 2D phase space from corner to corner.

To satisfy this uniform filling condition, the end points on the vertical phase-space boundary should be evenly distributed, i.e., the separation between two adjacent end points should be $2 \pi / N_{1}$. This can be achieved by making the measurement at $\lambda_{2}$ fill a $2 \pi$ range, i.e., $q_{2}=$ $2 \pi / L$. And even more generally, the condition is satisfied for all $q_{2}=2 \pi N_{2} / L$ as long as $N_{1}$ and $N_{2}$ are coprime, i.e., their greatest common divisor is 1 . This leads to the following criterion:

$$
\Delta q=2 \pi \Delta N / L
$$

where $\Delta q=\left|q_{1}-q_{2}\right|$ and $\Delta N=\left|N_{1}-N_{2}\right|$.

The formula above provides a list of candidate wavelengths that satisfy the uniform-filling condition. For a BGO voltage sensor with $\lambda_{1}=$ $1310 \mathrm{~nm}$ and a measurement range of $[-450 \mathrm{kV}$, $450 \mathrm{kV}]$, the list of the optimal second wavelengths is $\lambda_{2} \approx \lambda_{1}(1 \pm 0.0781 \Delta N) \in\{1215$ $\mathrm{nm}, 1421 \mathrm{~nm}, 1133 \mathrm{~nm}, 1553 \mathrm{~nm}, \cdots\}$. Since $1550 \mathrm{~nm}$ is a standard telecom wavelength at which many commercial components are available, it would be a particularly ideal choice for use in a dual-wavelength BGO voltage sensor.

\section{Summary}

We show that the unambiguous measurement range of an interferometric sensor can be extended to many phase periods, by combining two $2 \pi$-range phase shift measurements at distinct wavelengths. Compared with previous dual-wavelength techniques, our approach eliminates all measurement ambiguities, and is particularly suitable for an optical DC voltage sensor up to several 100s of kilovolts. Based on the mathematical properties of the signal, we have also developed a particularly efficient signal processing algorithm, which performs a fast 1D table lookup to convert the dualwavelength sensor output to the corresponding voltage value. A guideline for choosing the optimal sensor wavelengths for a given measurement range is also presented.

\section{References}

[1] G. A. Massey, D. C. Erickson, and R. A. Kadlec, "Electromagnetic field components: their measurement using linear electrooptic and magnetooptic effects," Applied Optics 14, 27122719 (1975)

[2] R. C. Miller, "Electro-optical voltage measuring system incorporating a method and apparatus to derive the measured voltage waveform from two phase shifted electrical signals," US Patent US4904931 (1990)
[3] R. C. Miller, "Method of deriving an AC waveform from two phase shifted electrical signals," US Patent US5001419 (1991)

[4] K. Kurosawa, S. Yoshida, E. Mori, G. Takahashi, and S. Saito, "Development of an optical instrument transformer for DC voltage measurement," IEEE Transactions on Power Delivery 8, 1721-1726 (1993)

[5] T. Bosselmann and O. Beierl, European Patent EP0864098B1 (1997)

[6] O. Beierl, T. Bosselmann, and M. Willsch, Patent W09805975A1 (1998)

[7] M. Stanimirov, U. Meier, K. Bohnert, and J. Glock, European Patent EP1179735A1 (2002)

[8] H. Lefèvre, The Fiber-Optic Gyroscope, Artech House (1993)

[9] K. Bohnert, P. Gabus, J. Nehring, and H. Brändle, "Temperature and vibration insensitive fiber-optic current sensor," Journal of Lightwave Technology 20, 267-276 (2002)

[10] K. Bohnert, P. Gabus, J. Nehring, H. Brändle, and M. G. Brunzel, "Fiber-Optic Current Sensor for Electrowinning of Metals," Journal of Lightwave Technology 25, 3602-3609 (2007) 